\title{
Implementación de sistemas de gestión integral sustentable para destinos turísticos, caso de estudio Parque Nacional Natural Utría, playa La Aguada - Colombia
}

\section{Fernando Gutiérrez-Fernández ${ }^{1}$ \& Natalia María Restrepo Sánchez ${ }^{2}$}

1. Docente Universidad El Bosque. Doctor por la Universidad Politécnica de Valencia en Desarrollo, Sostenibilidad y Ecodiseño, Colombia; gutierrezluisf@unbosque.edu.co

2. Docente Universidad El Bosque. Magister Gestión Ambiental Pontifica Universidad Javeriana, Colombia; restreponatis@unbosque.edu.co

Recibido: 13 de noviembre, 2014 - Aceptado: 11 de setiembre, 2015 - Corregido: 15 de octubre, 2015

\section{RESUMEN}

En el presente proyecto se diseñó e implementó un sistema de gestión integral sustentable para playa La Aguada, en tres niveles de gestión: estratégico, táctico y operacional; ya que se comparó dicha playa con una organización. Para el diseño del sistema, el primer paso fue determinar los requisitos establecidos en la NTS TS 001-2, que es la norma correspondiente en Colombia a la certificación de destinos turísticos de playa. Esta certificación busca mejorar, no solo las condiciones en el ámbito ambiental, sino que exista una transversalidad entre el componente ambiental, el socio-cultural, el económico y el de seguridad, contribuyendo al progreso y al mejoramiento de la calidad de vida de la comunidad anfitriona que se involucra directamente con la actividad del turismo. Playa La Aguada, se encuentra ubicada en el Parque Nacional Natural de Utría en el Departamento de Choco - Colombia y como destino turístico, tiene un principal atractivo que es el avistamiento de ballenas Jorobadas o Yubartas (Megaptera novaeangliae) Sin embargo, en los últimos años esta actividad se ha visto complementada por la realización de otro tipo de actividades eco turísticas, para lo cual la Unidad Administrativa Especial del Sistema de Parques Nacionales Naturales ha construido cabañas de alojamiento y un centro de visitantes. Por lo que esta se ha convertido en el lugar donde se recibe la mayor cantidad de visitantes, en el área natural y cuyo flujo se da durante todo el año; de manera que es necesario trabajar en su organización, buscando que el turismo no se convierta a futuro en una actividad perjudicial.

Palabras clave: Certificación de playas, NTS TS 001-2, playa La Aguada, turismo sostenible.

$$
\text { Formato de citación según APA }
$$

Gutiérrez Fernández, F. y Restrepo Sánchez, N.M. (2016). Implementación de sistemas de gestión integral sustentable para destinos turísticos, caso de estudio Parque Nacional Natural Utría, playa La Aguada - Colombia. Revista Espiga, Vol XV, (31), 13-29. 


\title{
ABSTRACT \\ Sustainable Integrated Management System Implementation for Tourist Destinations, study case in Utría Natural National Park, La Aguada beach-Colombia
}

\begin{abstract}
In the present project, a sustainable integrated management system was designed and implemented for La Aguada beach. For this purpose, three management levels are used: strategic, tactical and operational; since said beach was compared to an organization. For the system design, the first step was to determine the requirements of TS NTS 001-2, which is the norm in Colombia corresponding to certification of beach resorts. This certification aims to improve not only the conditions in the environmental field, but also the cross-cutting between the environmental, the economic, and security components; thus contributing to the progress and improvement of the quality of life of the hosting community directly involved with the tourist trade. Playa La Aguada is located in the Utría National Park, Departamento de Choco-Colombia. As a tourist destination, its main attraction is the sighting of humpback whales or Yubartas (Megaptera novaeangliae). However, in recent years, this activity has been complemented by performing other ecotourism activities, for which the Special Administrative Unit of National Parks has built lodging options and a visitor center. As a result, this beach represents the most visited place in the natural area. This flow of visitors can be seen throughout the year; so it is necessary to work in its organization, seeking that tourism does not turn out into a future harmful activity.
\end{abstract}

Key words: Beach certification, NTS TS 001-2, La Aguada beach, sustainable tourism.

\section{Introducción}

El turismo sostenible ya se encuentra definido por la Organización Mundial del turismo - OMT como: "El turismo que tiene plenamente en cuenta las repercusiones actuales y futuras, económicas, sociales y medioambientales para satisfacer las necesidades de los visitantes, de la industria, del entorno y de las comunidades anfitrionas". En Colombia, este concepto ha sido el elemento con el cual se desarrollaron las Normas Técnicas Sectoriales de Turismo Sostenible, entre las cuales se encuentra la 001-2 que corresponde a la de certificación de destinos turísticos de playa.

El proceso de implementación de sistemas de gestión de sostenibilidad y su posterior certificación, basado en las normas técnicas las cuales según Ochoa (Ochoa, 2008: 235), "son instrumentos para que el país alcance niveles de desarrollo y competitividad con el componente de la sostenibilidad", se debe dar en forma conjunta con las comunidades anfitrionas.

Estos esfuerzos se inscriben dentro del Plan Sectorial de Turismo de 2011- 2014 del Viceministerio de Turismo, Ministerio de Comercio, Industria y Turismo y el Departamento Nacional de Planeación, en el cual se afirma que en Colombia el turismo de sol y playa es el de mayor preferencia y posicionamiento, por lo que es importante contar con una estrategia de sostenibilidad que incluya la certificación en estas actividades turísticas del país. De igual forma, la Política de Playas Turísticas de 2011, afirma que "el crecimiento de la actividad turística no planificada, junto a la falta de un adecuado manejo de las playas, está generando la degradación de éstos recursos que en sí mismos, son la atracción turística, causando no solamente una pérdida económica sino una gran pérdida ecológica y cultural" (Ministerio de Comercio, Industria y Turismo, 2011: 9) En el mismo sentido se encuentra el Plan Sectorial de Turismo de 2014 -2018, cuyo objetivo estratégico es "Fomentar el desarrollo competitivo y sustentable de la industria de los viajes y el turismo, tanto en destinos, como en empresas del sector, a partir del aprovechamiento responsable de la diversidad natural y cultural, la inclusión diferenciada de comunidades étnicas, y la innovación en los productos $y$ servicios que se ofertan en las distintas regiones $y$ territorios de Colombia" (Ministerio de Comercio, Industria y Turismo, 2014: 39)

El presente estudio tuvo como objetivo principal establecer los lineamientos para la 
implementación de un sistema de gestión integral sustentable de playa La Aguada en el Parque Nacional Natural de Utría, en el departamento del Choco-Colombia, para su posterior certificación en turismo sostenible; resaltando la importancia del componente socio-cultural, ya que existe un traslape de lo que fue decretado como Área Nacional Protegida, con los resguardos y comunidades indígenas de la etnia Embera, ubicadas en la zona selvática y las comunidades negras que habitan el litoral (UAESPNN, 2009).

El establecimiento de estos lineamientos, con el apoyo de las entidades gubernamentales, se considera un facilitador en la toma de decisiones respecto al desarrollo del turismo y la conservación de los recursos naturales y el medio ambiente, lo cual representa finalmente la finalidad de las áreas protegidas.

Así mismo la implementación de lineamientos de sostenibilidad permite fortalecer las relaciones entre la comunidad anfitriona, la Fundación Mano Cambiada (operador turístico), los turistas y los funcionarios de la Unidad Administrativa Especial del Sistema de Parques Nacionales Naturales; ya que al haber un sistema de organización disminuyen los conflictos de intereses entre estos. De tal manera que se pueden desarrollar distintas actividades económicas, sin que estas sean motivo de conflicto comunitario y que se fomente la conservación y protección ambiental.

El propósito de la investigación fue innovar en el diseño de un Sistema de Gestión Integral Sustentable de un patrimonio ecoturístico y cultural ancestral de las comunidades indígenas y de la cultura afrocolombiana. El sistema diseñado e implementado pretende elevar el nivel de satisfacción del turista al ser receptor de un servicio de calidad y que a su vez garantice la conservación de los ecosistemas, mejorando la calidad de vida de las comunidades residentes, que se ven representadas por la Fundación Mano Cambiada. También el sistema pretende ser el elemento articulador en la recuperación de los valores culturales ancestrales y ecosistémicos. para lo cual, se comenzó identificando a la administración de la playa como una organización, y a esta como un sistema, en donde de acuerdo a los requisitos de la norma debe existir un líder de sostenibilidad, que hace las veces de gerente, y como tal debe asegurarse, que todas las partes interdependientes de la organización funcionen en conjunto para que ésta pueda alcanzar sus metas.

El sistema de gestión integral sustentable que se diseñó es específico de playa La Aguada, ya que como lo señala Gutiérrez-Fernández "las áreas naturales desde el punto de vista del turismo, tienen sus propias necesidades (...)" (GutiérrezFernández, 2013: 111)

Teniendo en cuenta lo anterior, se diseñó un sistema concebido en tres niveles: estratégico, táctico y operacional.

En el nivel estratégico, se tiene al nivel central de la UAESPNN (Jefe la Oficina de Sostenibilidad), como entidad que busca determinar cuáles son los negocios en los que la organización (Playa La Aguada) debe participar y cómo es el tema de la toma de decisiones.

En el nivel táctico se trabaja con la gerencia, específicamente con el líder de sostenibilidad, quien apoya al nivel estratégico. Su roles planificar, organizar, dirigir y controlar, el sistema de gestión integral sustentable. En este caso, se identificó al jefe del PNN Utría, que también tiene la tarea de realizar los planes de aprovisionamiento o solicitar los recursos necesarios al nivel central para el funcionamiento de la playa y su Sistema de Gestión.

En el nivel operativo, se tiene a los diferentes actores que llevan a cabo las tareas planificadas dentro de los programas y que realizan las actividades para el cumplimiento de las metas.

\section{Metodología}

Este estudio se desarrolló en 4 etapas principales, las cuales se muestran a continuación. En ella se utilizó la Investigación-Acción Participativa o IAP. Este es un método de estudio y acción de tipo cualitativo que busca obtener resultados fiables y útiles para mejorar situaciones colectivas--. El mismo basa la investigación en la participación de los propios colectivos a investigar. 
A. Realización del diagnóstico de los aspectos ambientales, socio-culturales, económicos y de seguridad de playa La Aguada para determinar el grado de cumplimiento actual de los requisitos del estándar de dicha zona.

B. Construcción participativa de escenarios de acción para el diseño de los lineamientos requeridos para la implementación del sistema de gestión para la sostenibilidad de playa Grande.

C. Diseño de los programas de mejora necesarios para el cumplimiento de los requisitos que propone la norma técnica sectorial, de acuerdo a los escenarios propuestos.

D. Construcción de los elementos del sistema de gestión que permitan desarrollar articuladamente los programas de los cuatro componentes (ambiental, económico, sociocultural y de seguridad) de forma sostenible en el tiempo.

\section{Resultados}

Según la metodología empleada y a la concepción del sistema de gestión para la sostenibilidad de playa La Aguada, a continuación se presentan los principales resultados obtenidos en cada una de las etapas.

\section{Construcción participativa de escenarios}

\section{Identificación de actores}

Esta etapa de la investigación se desarrolló durante 2 meses en el segundo semestre de 2012. En esta fecha se realizó un análisis de involucrados para identificar a los principales actores y los roles que estos deben ocupar dentro del sistema de gestión para la sostenibilidad de "playa La Aguada" y se obtuvo el siguiente resultado:

\section{Formulación conjunta de estrategias}

De acuerdo a los requisitos solicitados por la norma técnica (NTS TS 001-2) y el cumplimiento de los mismos en playa La Aguada, se establecieron, conjuntamente con los actores involucrados mencionados en la tabla 1, las estrategias, que a su vez se agruparon en 5 tipos:

1. Estrategia de delimitación territorial del destino turístico de playa

En el destino turístico de playa, de acuerdo a la norma técnica, se debe delimitar el área geográfica utilizando la cartografía básica oficial y la cartografía náutica oficial a una escala adecuada y se debe diseñar, implementar y hacer seguimiento a una zonificación, uso y actividades de la playa, teniendo en cuenta la reglamentación vigente que le es aplicable por su naturaleza. Por lo que se diseñó un programa para llevar a cabo lo mencionado anteriormente.

a. Programa Delimitación y zonificación de playa La Aguda

2. Estrategia ambiental

Dentro de la norma técnica el componente ambiental presenta una elevada importanciaPor lo tanto, es necesario desarrollar varios programas para poder dar solución a todos los requisitos que contiene la norma de sostenibilidad y que buscan la calidad ambiental de los destinos de playa. Dentro de la estrategia ambiental se encuentran 10 programas que se mencionan a continuación.

a. Programa para minimizar los impactos negativos generados en la playa

b. Programa para monitorear la calidad de agua de baño y mantenerla

c. Programa para monitorear la calidad del material constitutivo de la playa y mantenerla

d. Programa uso eficiente del agua

e. Programa de agua potable

f. Programa de uso eficiente de la energía prevención

g. Programa de control de impactos atmosféricos

h. Programa de control de impactos visuales

i. Programa de control de impactos auditivos 
TABLA 1

Clasificación de actores

\begin{tabular}{|c|c|c|c|c|}
\hline Nivel en el S.G.S. & $\begin{array}{l}\text { Clasificación } \\
\text { de actores }\end{array}$ & $\begin{array}{l}\text { Nombre } \\
\text { del actor }\end{array}$ & $\begin{array}{c}\text { Intereses u objetivos } \\
\text { del actor }\end{array}$ & $\begin{array}{l}\text { Problemas } \\
\text { percibidos }\end{array}$ \\
\hline Estratégico & $\begin{array}{l}\text { Unidad Administrativa } \\
\text { Especial del Sistema } \\
\text { de Parques Naturales } \\
\text { Nacionales }\end{array}$ & $\begin{array}{l}\text { Jefe Oficina de } \\
\text { Sostenibilidad }\end{array}$ & $\begin{array}{l}\text { Encontrar la sostenibili- } \\
\text { dad financiera }\end{array}$ & Bajos ingresos del área \\
\hline Táctico & $\begin{array}{l}\text { Funcionarios del PNN } \\
\text { Utría }\end{array}$ & Jefe del PNN Utría & $\begin{array}{l}\text { Mantener la conservación } \\
\text { del PNN Utría }\end{array}$ & $\begin{array}{l}\text { Bajos recursos asignados } \\
\text { por el nivel central }\end{array}$ \\
\hline \multirow[t]{6}{*}{ Operacional } & \multirow[t]{6}{*}{$\begin{array}{l}\text { Usuarios de recursos } \\
\text { naturales; (nativos, } \\
\text { pobladores, asociaciones, } \\
\text { pescadores, entre otros) }\end{array}$} & $\begin{array}{l}\text { Comunidades } \\
\text { indígenas } \\
\text { Comunidades negras }\end{array}$ & $\begin{array}{l}\text { Participar de los ingresos } \\
\text { del turismo } \\
\text { Participar de los ingresos } \\
\text { del turismo }\end{array}$ & $\begin{array}{l}\text { Pérdida de su cultura y } \\
\text { deterioro del ecosistema } \\
\text { Pérdida de su cultura y } \\
\text { deterioro del ecosistema }\end{array}$ \\
\hline & & Lancheros & $\begin{array}{l}\text { Mantenimiento del turis- } \\
\text { mo en la playa para mayo- } \\
\text { res ingresos económicos/ } \\
\text { Prestación de un buen } \\
\text { servicio a los turistas }\end{array}$ & $\begin{array}{l}\text { No hay un sitio de } \\
\text { parqueo determinado de } \\
\text { las lanchas en la playa/ } \\
\text { En algunos casos exceden } \\
\text { el número de pasajeros } \\
\text { permitidos y no aseguran } \\
\text { el cumplimiento del uso de } \\
\text { salvavidas. }\end{array}$ \\
\hline & & Pescadores & $\begin{array}{l}\text { Que se conserven los } \\
\text { peces para asegurar su } \\
\text { subsistencia/ Conservar la } \\
\text { tradición }\end{array}$ & $\begin{array}{l}\text { Muchas veces el turismo } \\
\text { irrumpe sus actividades } \\
\text { cotidianas, ya que algunas } \\
\text { playas para la pesca } \\
\text { son aledañas a playa La } \\
\text { Aguada }\end{array}$ \\
\hline & & $\begin{array}{l}\text { Asociación Mano } \\
\text { Cambiada }\end{array}$ & $\begin{array}{l}\text { Aumentar el ingreso de } \\
\text { turistas y la venta de } \\
\text { alimentos }\end{array}$ & $\begin{array}{l}\text { Bajo nivel de turistas / } \\
\text { Ocupación alta en tempo- } \\
\text { rada de ballenas }\end{array}$ \\
\hline & & Turistas & $\begin{array}{l}\text { Conocer y disfrutar el } \\
\text { ecosistema }\end{array}$ & $\begin{array}{l}\text { No tienen conocimiento } \\
\text { sobre prácticas sostenibles } \\
\text { en turismo/En algunos ca- } \\
\text { sos, no hay un buen trato } \\
\text { de los turistas hacia los } \\
\text { prestadores de servicios }\end{array}$ \\
\hline & & $\begin{array}{l}\text { Funcionarios } \\
\text { del PNN Utría }\end{array}$ & $\begin{array}{l}\text { Asegurar la conservación } \\
\text { del PNN }\end{array}$ & $\begin{array}{l}\text { Escasos recursos tec- } \\
\text { nológicos, humanos y } \\
\text { económicos }\end{array}$ \\
\hline
\end{tabular}

Fuente: elaboración propia. 
j. Programa para evitar la introducción de residuos sólidos a la playa.

\section{Estrategia sociocultural}

Esta estrategia busca gestionar todos los impactos socioculturales, entendiendo estos como las transformaciones positivas o negativas que produce el desarrollo de actividades turísticas sobre el patrimonio cultural; tradiciones, costumbres y formas de vida de las comunidades locales y sitios de interés histórico y cultural en un destino turístico de playa. Para el desarrollo de la estrategia se formularon 3 programas.

a. Programa de información y sensibilización para turistas y prestadores de servicios turísticos, sobre buenas prácticas en la playa.

b. Programa para el reconocimiento, la apropiación y la promoción del patrimonio cultural local.

c. Programa de capacitación de actores involucrados sobre conceptos y criterios de calidad y sostenibilidad de las playas dirigida.

4. Estrategia económica

Esta estrategia busca gestionar todos los impactos económicos, entendiéndolos como las transformaciones positivas o negativas que produce el desarrollo de actividades turísticas en las dinámicas económicas del destino turístico de playa, para lo cual se diseñó un programa para medir el grado de satisfacción de los visitantes y turistas a la playa y de acuerdo con el análisis de los resultados formular e implementar acciones para mejorar la misma.

\section{Estrategia de seguridad}

6. Dentro de la estrategia de seguridad se encuentra el conjunto de programas que se aplican para atender la seguridad de los turistas y la comunidad local en un destino turístico de playa. Para el caso especifico de La
Aguada, se formularon dos programas en los que se encuentran las responsabilidades, recursos, medios y plazos para la ejecución de la estrategia y que se citan a continuación:

a. Programa de seguridad y señalización

b. Programa de prevención de emergencias y contingencias

\section{Análisis de roles de los actores involucrados}

Para determinar el rol de cada uno de los actores identificados, se diseñó una tabla, en donde de forma participativa, se iban identificando los programas y la participación de los diferentes grupos. Ver tabla 2.

\section{Diseño de programas}

\section{Organización y análisis de estrategias y programas}

Durante los meses de noviembre y diciembre de 2012 se realizó una mesa de trabajo con los actores involucrados en la gestión de playa La Aguada, con el fin de definir qué programas de las 5 estrategias, se encontraban relacionados entre sí; para lo cual se definieron dos escalas de relación débil y fuerte.

Lo anterior arrojó que uno de los programas con mayor sinergia con los otros es el de información y sensibilización para turistas y prestadores de servicios turísticos, sobre buenas prácticas y buen uso de la playa por parte de los visitantes y turistas. Este mostró una relación fuerte con 8 de los 10 programas de la estrategia ambiental.

Igualmente, se pudo evidenciar que el programa para minimizar los impactos negativos generados en la playa se relaciona débilmente con 8 de los programas de la misma estrategia ambiental y con el programa de Delimitación y Zonificación de playa La Aguda, de la estrategia de delimitación territorial del destino turístico de la playa.

Lo anterior permitió que se formularan las actividades de los programas de tal forma que se fortalezcan los programas que se relacionan entre sí. 
TABLA 2

Estrategias y actores involucrados

\begin{tabular}{|c|c|c|c|}
\hline Estrategia & Programa & Líder del programa & Involucrados en el programa \\
\hline $\begin{array}{l}\text { Delimitación terri- } \\
\text { torial del destino } \\
\text { turístico de playa }\end{array}$ & $\begin{array}{l}\text { Delimitación y zonificación de playa La } \\
\text { Aguda }\end{array}$ & Jefe del PNN Utría & $\begin{array}{l}\text { Funcionarios del PNN Utría } \\
\text { Lancheros } \\
\text { Pescadores }\end{array}$ \\
\hline \multirow[t]{10}{*}{$\begin{array}{l}\text { Estrategia } \\
\text { ambiental }\end{array}$} & $\begin{array}{l}\text { Programa para minimizar los impactos negati- } \\
\text { vos generados en la playa. }\end{array}$ & $\begin{array}{l}\text { Funcionarios del PNN } \\
\text { Utría }\end{array}$ & $\begin{array}{l}\text { Comunidades negras } \\
\text { Lancheros } \\
\text { Pescadores } \\
\text { Asociación Mano Cambiada } \\
\text { Turistas }\end{array}$ \\
\hline & $\begin{array}{l}\text { Programa para monitorear la calidad de agua } \\
\text { de baño y mantenerla }\end{array}$ & Jefe del PNN Utría & Funcionarios del PNN Utría \\
\hline & $\begin{array}{l}\text { Programa para monitorear la calidad del mate- } \\
\text { rial constitutivo de la playa y mantenerla }\end{array}$ & Jefe del PNN Utría & Funcionarios del PNN Utría \\
\hline & Programa uso eficiente del agua & $\begin{array}{l}\text { Asociación Mano } \\
\text { Cambiada }\end{array}$ & Turistas \\
\hline & Programa de agua potable & $\begin{array}{l}\text { Asociación Mano } \\
\text { Cambiada }\end{array}$ & Turistas \\
\hline & Programa de uso eficiente de la energía & $\begin{array}{l}\text { Asociación Mano } \\
\text { Cambiada }\end{array}$ & Turistas \\
\hline & Programa de control de impactos atmosféricos & $\begin{array}{l}\text { Funcionarios del PNN } \\
\text { Utría }\end{array}$ & $\begin{array}{l}\text { Comunidades negras } \\
\text { Lancheros } \\
\text { Pescadores } \\
\text { Asociación Mano Cambiada } \\
\text { Turistas }\end{array}$ \\
\hline & Programa de control de impactos visuales & $\begin{array}{l}\text { Funcionarios del PNN } \\
\text { Utría }\end{array}$ & $\begin{array}{l}\text { Asociación Mano Cambiada } \\
\text { Turistas }\end{array}$ \\
\hline & Programa de control de impactos auditivos & $\begin{array}{l}\text { Asociación Mano } \\
\text { Cambiada }\end{array}$ & $\begin{array}{l}\text { Lancheros } \\
\text { Pescadores } \\
\text { Turistas }\end{array}$ \\
\hline & $\begin{array}{l}\text { Programa para evitar la introducción de resi- } \\
\text { duos sólidos a la playa }\end{array}$ & $\begin{array}{l}\text { Asociación Mano } \\
\text { Cambiada }\end{array}$ & $\begin{array}{l}\text { Lancheros } \\
\text { Pescadores } \\
\text { Turistas }\end{array}$ \\
\hline \multirow[t]{3}{*}{$\begin{array}{l}\text { Estrategia } \\
\text { sociocultural }\end{array}$} & $\begin{array}{l}\text { Programa de información y sensibilización } \\
\text { para turistas y prestadores de servicios turís- } \\
\text { ticos, sobre buenas prácticas buen uso de la } \\
\text { playa por parte de los visitantes y turistas. }\end{array}$ & $\begin{array}{l}\text { Funcionarios del PNN } \\
\text { Utría }\end{array}$ & $\begin{array}{l}\text { Asociación Mano Cambiada } \\
\text { Turistas }\end{array}$ \\
\hline & $\begin{array}{l}\text { Programa para el reconocimiento, la apropia- } \\
\text { ción y la promoción del patrimonio cultural } \\
\text { local. }\end{array}$ & $\begin{array}{l}\text { Funcionarios del PNN } \\
\text { Utría }\end{array}$ & $\begin{array}{l}\text { Comunidades negras } \\
\text { Lancheros } \\
\text { Pescadores } \\
\text { Asociación Mano Cambiada } \\
\text { Turistas }\end{array}$ \\
\hline & $\begin{array}{l}\text { Programa de capacitación de actores involu- } \\
\text { crados sobre conceptos y criterios de calidad y } \\
\text { sostenibilidad de las playas }\end{array}$ & $\begin{array}{l}\text { Funcionarios del PNN } \\
\text { Utría }\end{array}$ & $\begin{array}{l}\text { Comunidades negras } \\
\text { Lancheros } \\
\text { Pescadores } \\
\text { Asociación Mano Cambiada } \\
\text { Turistas }\end{array}$ \\
\hline
\end{tabular}


TABLA 2 (Continuación)

Estrategias y actores involucrados

\begin{tabular}{llll}
\multicolumn{1}{c}{ Estrategia } & \multicolumn{1}{c}{ Programa } & \multicolumn{1}{c}{ Líder del programa } & \multicolumn{1}{c}{ Involucrados en el programa } \\
$\begin{array}{l}\text { Estrategia } \\
\text { económica }\end{array}$ & $\begin{array}{l}\text { Programa para medir el grado de satisfacción } \\
\text { de los visitantes y turistas de la playa y de } \\
\text { acuerdo con el análisis de los resultados for- } \\
\text { mular e implementar acciones para mejorar. }\end{array}$ & Funcionarios del PNN & $\begin{array}{l}\text { Lancheros } \\
\text { Asociación Mano Cambiada } \\
\text { Turistas }\end{array}$ \\
\hline $\begin{array}{l}\text { Estrategia de } \\
\text { seguridad }\end{array}$ & Programa de seguridad y señalización & Jefe del PNN Utría & Funcionarios del PNN Utría \\
\hline
\end{tabular}

\section{Diseño de los programas}

\section{Estrategia delimitación territorial del destino turístico de playa}

\section{Delimitación y zonificación de playa La Aguada}

La playa presenta dos tipos de uso, la zona de alta densidad de uso, que comprende las cabañas de alojamiento, el restaurante Yubarta y la cabaña de los funcionarios de parques, y la zona de recreación general exterior, que es la playa como tal.

La zona de recreación general exterior (playa La Ensenada - La Aguada) va desde el punto de Estero Grande, ubicado en las coordenadas N 6 01'36.6 y W 77 20'88,0" y el río Aguada ubicado en las coordenadas N 600 ' 72.3" y W 7720 ' 66.5 ", con una distancia de $1.25 \mathrm{~km}$. Se calculó que durante la marea baja se cuenta con un área de uso seguro de 50 metros aproximadamente de ancho. Para el cálculo total de área de uso se estimó la distancia en la zona de playa de $1.25 \mathrm{~km}$, una distancia medida en la parte del bosque (zona de servicios turísticos) de $1.26 \mathrm{~km}$ y en total un área aproximada de $63.582 \mathrm{~m}^{2}$.

En la parte de playa la Ensenada, donde se encuentran corales, está prohibido el ingreso de bañistas, o de la práctica de actividades de buceo o careteo (snorkeling) Esta zona se encuentra frente a la cabaña de parques nacionales y está demarcada por unas boyas de color rojo.

\section{Zonificación playa La Aguada}

- Zona de servicios turísticos

Esta zona cuenta con las siguientes instalaciones: cabaña Jaibana, restaurante Yubarta, cabaña Guayabillo, cabaña Dormilón, auditorio.

- Zona de enlace

Entre la cabaña Jaibana - Restaurante Yubarta y Puente, se encuentra una zona que cuenta con jardines y área para la movilidad peatonal. Así mismo, cuenta con una ducha de agua dulce que utilizan los bañistas de pasadía, ya que las cabañas cuentan con grifos de agua dulce en la entrada para el lavado de los pies.

\section{- Zona de reposo}

Es la zona donde empieza el material constitutivo de la playa (arena) y como se dijo anteriormente esta es de un ancho aproximado de 50 metros durante la marea baja, ya que durante la marea alta esta zona desaparece casi en su totalidad. Esta es la zona conocida como playa La Aguada.

\section{- Zona de bañistas}

Se puede utilizar la playa desde Estero Grande hasta la zona donde empiezan los corales, la cual está demarcada por una boya roja. Sin embargo, se recomienda utilizar únicamente la zona frente a la cabaña Jaibana y restaurante Yubarta, hasta donde se encuentra una plataforma de 
observación de peces y que además muestra el sitio donde se encuentra un barco hundido.

Esto a su vez permite que exista una mayor seguridad y que el bañista no entre en contacto con las embarcaciones.

- Zona deportes náuticos

No está permitida la práctica de deportes náuticos con motor, ya que se presenta tránsito de mamíferos acuáticos. Se permite el canotaje, careteo y el buceo excluyendo las zonas de corales.

\section{Estrategia ambiental}

\section{Programa para minimizar los impactos negativos generados en la playa}

Para el diseño de la estrategia de sostenibilidad y minimizar los impactos negativos generados en la playa en estudio, se realizó una mesa de trabajo con los actores involucrados para poder construir la línea de base. En esta se realizó la identificación de actividades turísticas y sus respetivos impactos. Para lo cual se elaboró una matriz que da cuenta del proceso por dimensión (ambiental, sociocultural y económica), donde se muestran los diferentes impactos de sostenibilidad producidos en la zona en cuestión por las diferentes actividades que allí se originan, siendo el puntaje mínimo posible de 9 y el máximo de 45 (ver anexo1) En la tabla No. 2 se muestran los principales impactos obtenidos.

El diligenciamiento de esta matriz se realizó de igual forma de manera participativa con los actores que tienen injerencia en la playa. De forma que cada uno, de acuerdo con su experiencia y conocimientos específicos de su actividad, identificó los impactos y las acciones para mitigarlos.

A continuación, se muestran los principales resultados de la matriz diligenciada:

TABLA 3

Principales impactos zona de la playa La Aguada.

\begin{tabular}{lcc}
$\quad$ Actividad & Carácter del impacto & Calificación \\
Senderismo submarino (Buceo) & Negativo & 15 \\
Investigación & Negativo & 15 \\
Educación Ambiental & Positivo & 21 \\
Adquisición de Artesanías (Esta actividad no se & Negativo & 15 \\
realiza en la actualidad, pero se proyecta a futuro) & & \\
Ingreso a la playa en embarcaciones & Negativo & 17 \\
\hline
\end{tabular}

Fuente: elaboración propia.

\section{Programa para monitorear la calidad de agua de baño y mantenerla}

En Colombia no existe una normativa que regule la calidad del agua de baño. Sin embargo, a nivel internacional se encuentra legislación respecto al tema. Por ejemplo en España, donde se regula la calidad de las aguas de baño mediante el Real Decreto 734/1988, del 1 de julio, por el que se establecen las normas de calidad para las aguas de baño y en la Directiva 2006/7/CE, del Parlamento Europeo y del Consejo del 15 de febrero, relativa a la gestión de la calidad de las aguas de baño.

Se define "aguas de baño" como "cualquier elemento de aguas superficiales en el que las autoridades competentes prevean que se bañe un número importante de personas y en el que no exista una prohibición permanente de baño ni se haya formulado una recomendación permanente de abstenerse del mismo", NTS TS 2011.

En este sentido, y para preservar la calificación de aguas para baño, es necesario controlar y clasificar convenientemente la calidad 
de las aguas destinadas para tal fin, gestionar su calidad y suministrar información sobre calidad a la población.

La estrategia para monitorear la calidad de agua de baño responde a lo establecido en la norma NTC-ISO 5667-9.

\section{Resultados}

La muestra de agua de baño se recogió de acuerdo a lo establecido en el procedimiento y entregada al laboratorio el 14 de noviembre de 2012. El análisis fue efectuado el 15 de noviembre de 2012.

- $\mathrm{pH}=6.9$

- $\quad$ Salmonella spp = N.D

- $\quad$ Coliformes totales $=3.3 \mathrm{E}+00$

- Coliformes fecales $=<0.18$

\section{Programa para monitorear la calidad del material constitutivo de la playa y mantenerla}

El aporte directo y continuo de contaminantes de origen orgánico e inorgánico no solo daña el agua de los mares, sino también afecta la calidad de los sedimentos y con ello el material constitutivo de la playa.

Por lo anterior, se diseñó el programa de monitoreo de la calidad del material constitutivo de la playa en las mismas fechas que se realice el muestreo del agua de mar. Se recomienda realizar un análisis de los elementos totales del material constitutivo de la playa mínimo dos veces al año. fueron:

Las metodologías de laboratorio utilizadas

- Para salmonella spp, se realizó recuento en placa

- Para coliformes fecales y totales: se empleó el número más probable, que es un método que se fundamenta en la capacidad de este grupo microbiano de fermentar la lactosa con producción de ácido y gas al incubarlos a $35^{\circ} \mathrm{C} \pm 1{ }^{\circ} \mathrm{C}$ durante 48 horas, utilizando un medio de cultivo que contenga sales biliares. Esta determinación consta de dos fases: la fase presuntiva y la fase confirmativa. En la fase presuntiva el medio de cultivo que se utiliza es el caldo lauril sulfato de sodio, el cual permite la recuperación de los microorganismos dañados que se encuentren presentes en la muestra y que sean capaces de utilizar a la lactosa como fuente de carbono. Durante la fase confirmativa se emplea como medio de cultivo caldo lactosado bilis verde brillante, el cual es selectivo y solo permite el desarrollo de aquellos microorganismos capaces de tolerar tanto las sales biliares como el verde brillante.

- $\mathrm{pH}$ : potenciometria en agua, muestra fresca.

- Calcio, magnesio, potasio, sodio, fosforo, manganeso, hierro, zinc, cobre, sílice, aluminio, azufre, boro y cromo: digestión en mezcla acida en microondas.

- Calcio, magnesio, hierro, manganeso, zinc, cobre, aluminio, silicio y cromo: cuantificación por absorción atómica.

- Potasio y sodio: emisión atómica.

\section{Resultados}

El día 11 de noviembre de 2012, se recolectó una muestra de aproximadamente un kilo de material constitutivo de playa, se almacenó en una bolsa de polietileno de baja densidad y fue guardada en nevera para su traslado al laboratorio ${ }^{1}$. El muestreo se realizó de forma manual empleando un tubo de PVC para la recolección de la muestra.

Los resultados fueron los siguientes:

- $\mathrm{Ph}=7.2$

- $\quad$ Salmonella $=$ N.D

1. Esta metodología ha sido usada en estudios como CARACTERIZACIÓN QUÍMICA DE LOS SEDIMENTOS SUMERGIDOS, ELEMENTO CLAVE DENTRO DE UN PROGRAMA DE MONITOREO AMBIENTAL INTEGRAL EN LAS PLAYAS DE LA BAHÍA DE MATANZAS; realizado por M. Sc. Mariela Almeida Rodríguez, M. Sc. Yohandra de Armas Vargas y Téc. Lilian Rojas López. 
- Coliformes Totales (NMP/g o ml de muestra) $=<1.8$

- Coliformes Fecales (NMP/g o ml de muestra) $=<1.8$

- Calcio $(\mathrm{Ca})=11.75 \%$

- Oxido de Calcio $(\mathrm{CaO})=16.45 \%$

- Magnesio $(\mathrm{Mg})=1.06 \%$

- Oxido de Magnesio $(\mathrm{MgO})=1.76 \%$

- $\quad$ Potasio $(\mathrm{K})=0.03 \%$

- Oxido de Potasio $\left(\mathrm{K}_{2} \mathrm{O}\right)=0.04 \%$

- $\quad$ Sodio $(\mathrm{Na})=0.18 \%$

- Oxido de sodio $\left(\mathrm{Na}_{2} \mathrm{O}\right)=0.24 \%$

- Aluminio $(\mathrm{Al})=8.23 \%$

- Oxido de aluminio $\left(\mathrm{Al}_{2} \mathrm{O}_{2}\right)=15.55 \%$

- $\quad$ Silicio $(\mathrm{Si})=15.08 \%$

- Oxido de silicio $\left(\mathrm{SiO}_{2}\right)=32,27 \%$

- Hierro $(\mathrm{Fe})=22.43 \%$

- Óxido de hierro $\left(\mathrm{Fe}_{2} \mathrm{O}_{3}\right)=32.07 \%$

- Magnesio $(\mathrm{mn})=1530(\mathrm{mg} / \mathrm{kg})$

- Óxido de magnesio $(\mathrm{MnO})=1974(\mathrm{mg} / \mathrm{kg})$

- $\quad \operatorname{Zinc}(\mathrm{Zn})=230(\mathrm{mg} / \mathrm{kg})$

- Óxido de Zinc $(\mathrm{ZnO})=285(\mathrm{mg} / \mathrm{kg})$

- $\quad$ Cobre $(\mathrm{Cu})=0.01(\mathrm{mg} / \mathrm{kg})$

- Óxido de Cobre $(\mathrm{CuO})=0.01$

\section{Programa uso eficiente del agua}

El agua que se utiliza proviene del río $\mathrm{La}$ Aguada, el cual es perenne o permanente, ya que presenta escasas fluctuaciones a lo largo del año. No se cuenta con medidores de consumo, dado que el recurso se toma directamente de la fuente natural.

Se cuenta con un desarenador de flujo horizontal en la parte alta del río, el cual es una estructura hidráulica que tiene como función remover las partículas de cierto tamaño que la captación de una fuente superficial permite pasar. Las partículas se sedimentan al reducirse la velocidad con que son transportadas por el agua.

El agua sale del desarenador y es transportada hasta la zona de alojamiento y a la cabaña de los funcionarios de parques mediante una tubería. Sin embargo, en ocasiones esta sufre roturas dada la presión que debe soportar. Por lo tanto, teniendo en cuenta que el recurso hídrico con el cual se abastece la playa "La Ensenada" no es una limitante, la estrategia se centrará en generar conciencia a los visitantes. A ellos se les informará que si bien el recurso no es una limitante, su uso en los alojamientos y en los baños implica que se generen vertimientos, por lo que se realizará énfasis en el programa de información y sensibilización para turistas y prestadores de servicios turísticos, sobre buenas prácticas.

\section{Programa de agua potable}

El prestador de servicios ecoturísticos "Mano cambiada" tiene agua potable en envases plásticos. Sin embargo, con el fin de garantizar una mayor disponibilidad del recurso y ayudar a la estrategia de residuos sólidos y disminuir la presencia de envases plásticos, se contrató al Laboratorio Nacional de Suelos del Instituto Geográfico Agustín Codazzi, para realizar un análisis de potabilidad de una muestra de agua tomada en el restaurante Yubarta, previamente tratado por el filtro con el que cuenta el lugar.

El día 14 de noviembre de 2012, se llevó al laboratorio, habiendo observado todos los parámetros establecidos en la NORMA TÉCNICA NTC-ISO COLOMBIANA 5667-3, de fecha 2004-11-03, en donde se establecen las directrices para la preservación y manejo de las muestras.

Los resultados muestran que los niveles de Coliformes fecales se encuentran en concentraciones no permitidas para el consumo humano. Por lo que como programa para potabilizar el agua, a mediano plazo se propone la adquisición de un ozonificador para tratar el agua en el restaurante Yubarta y volver a analizar su potabilidad.

No obstante lo anterior, se señala la disponibilidad en el momento de agua potable envasada de marcas comerciales.

\section{Programa de uso eficiente de la energía}

Las instalaciones de las cabañas de alojamiento no poseen energía eléctrica, se está estudiando la viabilidad de dotarlas con energía fotovoltaica. 
En el Restaurante Yubarta y la cabaña de los funcionarios de parques se cuenta con paneles solares que abastecen de energía. Este tipo de electricidad es renovable y es obtenida directamente a partir de la radiación solar.

Cabe anotar que la posibilidad de generar energía con paneles solares en la región pacífica a la cual pertenece la playa "La Ensenada" es de las más bajas del país, de acuerdo al atlas de radiación solar de Colombia, elaborado por la UPME (ver tabla 4)

TABLA 4

Potencialidad de generación con paneles solares.

\begin{tabular}{lc} 
& $\mathrm{kWh} / \mathrm{m}^{2} / \mathrm{año}$ \\
Guajira & 2.190 \\
Costa atlántica & 1.825 \\
Orinoquia & 1.643 \\
Amazonia & 1.551 \\
Andina & 1.643 \\
Costa pacífica & 1.278 \\
\hline
\end{tabular}

Fuente: UPME, 2005.

No obstante lo anterior, esta energía tiene ventajas como las que describe el Instituto de Planificación y Promoción de Soluciones Energéticas para los Zonas no Interconectadas - IPSE:

VENTAJAS: cuenta con energía no contaminante, inagotable, sistema idóneo para zonas no interconectadas, de fácil mantenimiento, el costo disminuye a medida que la tecnología va avanzando.

Por lo anterior, y teniendo en cuenta que la energía que actualmente se utiliza es de fuentes renovables y es limpia, la estrategia se basa en divulgar buenas prácticas de uso energético, con las cuales se pueda garantizar que en días de poca radiación solar exista la posibilidad de contar con electricidad para las necesidades básicas.

\section{Programa de control de impactos atmosféricos}

Dado que no existen fuentes de impactos atmosféricos por maquinaria, se realiza únicamente las siguientes dos indicaciones:
- Las fogatas no están permitidas

- Reemplazar las botellas de gasolina que se usan para iluminar el sendero en las noches

\section{Programa de control de impactos visuales}

- Se realizan las siguientes dos indicaciones:

- No se permite extender toallas, ni ropa en los balcones de las cabañas.

- Retirar publicidad de la asociación Mano Cambiada y de eventos realizados en el PNN Utría

\section{Programa de control de impactos auditivos}

El uso de radios, grabadoras aparatos de música con volumen alto que pudiera perturbar el ambiente de la playa "La Aguada" está prohibido con excepción de los eventos autorizados por la UAESPNN.

\section{Programa para evitar la introducción de residuos sólidos a la playa}

Para lograr formular una estrategia idónea al entorno de playa La Aguada, se realizó la identificación y diagnóstico en cuanto a los puntos de generación de basuras y residuos sólidos que se localizan dentro de la playa. Estos son las 4 cabañas de operación turística (Jaibana, Guayabillo, Dormilon y Restaurante Yubarta), la cabaña de PNN, zona de resguardo del pelotón de Infantería de Marina y el Auditorio Utría - Manglar, adicionalmente a estos puntos cabe mencionar la llamada "Puja de San Pacho" que es el nombre que le dan los nativos a la marea más alta y que trae consigo una cantidad considerable de residuos que quedan en parte de la playa La Aguada

De acuerdo a lo anterior se proponen las siguientes actividades:

- Realizar una charla cada vez que ingresan turistas al Parque, en la cual se den al visitante recomendaciones para que no arroje basuras en el área y se devuelvan con ella al finalizar su visita, ya que los puntos ecológicos que 
se encuentran ubicados en el Restaurante, Oficina de Parques y en la construcción del auditorio tienen como fin la separación en la fuente que generan los funcionarios de PNN y de Mano Cambiada.

- Construir un cuarto de almacenamiento temporal.

- Compostar los residuos orgánicos que constituyen el mayor porcentaje de residuos generados en el Parque.

- Comprar los alimentos y productos de aseo al por mayor y no por unidades.

- Con las botellas de PET se comenzó a realizar una actividad que consiste en rellenarlas por dentro con empaques de pasabocas para la decoración del jardín nativo que se tiene en el área de alojamiento

\section{Estrategia sociocultural}

Programa de información y sensibilización para turistas y prestadores de servicios turísticos, sobre buenas prácticas y buen uso de la playa

Se realizó una cartilla de buenas prácticas, que se da a conocer al ingreso del visitante, así como un video divulgativo de los diferentes programas y comportamientos que se deben adoptar al interior del PNN Utría y cuando se realiza uso de la playa La Aguada.

Programa para el reconocimiento, la apropiación y la promoción del patrimonio cultural local

Se va a ofrecer gastronomía típica de la región, entre la que se encuentra el arroz de Piangua, mariscadas y peces de temporada, este programa se basa en la promoción de las recetas de la zona.

Programa de capacitación de actores involucrados sobre conceptos y criterios de calidad y sostenibilidad de las playas dirigida

Se capacitó a los actores involucrados y se les dio un curso sobre los principios y los requisitos de las normas técnicas sectoriales de turismo sostenible.

\section{Estrategia económica}

Programa para medir el grado de satisfacción de los visitantes y turistas a la playa y de acuerdo con el análisis de los resultados formular e implementar acciones para mejorar.

Se tiene previsto realizar encuestas para medir el grado de satisfacción de los visitantes, y analizarlas de forma mensual con la finalidad de implementar programas de mejora, si la calificación de la satisfacción decrece.

\section{Estrategia de seguridad}

\section{Programa de seguridad y señalización}

Las principales amenazas que se presentan en la zona, se tomaron de un documento interno de trabajo del PNN Utría en donde se determinó el nivel de riesgo, de acuerdo a la siguiente formula, RIESGO = AMENAZA + VULNERABILIDAD. Cabe aclarar que para los riesgos, que el área natural identificó con relación al conflicto interno colombiano, no se desarrolló ningún plan de acción, únicamente se trabajó en los riesgos de origen natural. Dado que el riesgo de Tsunami se determinó como elevado, se realizó la adecuación de la ruta de evacuación, la cual parte del centro de visitantes Jaibana hasta el punto de encuentro en la cabaña de funcionarios. Posteriormente, se sigue por la playa, se sube por la quebrada La Aguada, se sube hacia la loma La Aguada y se llega hasta la zona segura a 75 metros de altura. El recorrido se realiza en un promedio de $20 \mathrm{mi}-$ nutos sin correr.

\section{Construcción de los elementos de sistema de gestión que permitan desarrollar articuladamente los programas de los cuatro componentes (ambiental, económico, sociocultural y de seguridad) de forma sostenible en el tiempo.}

Como se mencionó en la introducción se diseñó un sistema de gestión para la sostenibilidad, equiparando a la playa, con una organización, por lo que el sistema se propuso en tres niveles estratégico, táctico y operacional. 
De igual forma se definió que los canales de divulgación debían conservar los tres niveles. Por lo que si un programa no funciona adecuadamente, debe ser el líder del mismo, el que informe al nivel táctico y si este no puede dar solución, se recurre al siguiente nivel (estratégico).

Por último, se diseñaron los formatos para recabar las evidencias del funcionamiento del sistema y se realizó su codificación y su tabla de retención documental.

\section{Discusión de resultados}

Fue necesario diseñar un sistema de gestión integral sustentable para playa La Aguada ubicada en el PNN Utría, en tres niveles de gestión: estratégico, táctico y operacional, con el fin de garantizar la sostenibilidad del propio sistema y no solo el cumplimiento de los requisitos establecidos en la norma técnica sectorial de turismo sostenible.

En el nivel operacional se formularon los programas para operar los componentes (estrategias) ambiental, sociocultural, económico y de seguridad, y se proyectaron las actividades necesarias para dar cumplimiento a los requisitos de la norma técnica y superar las falencias en el cumplimiento de parámetros que afectan la prestación del servicio y el producto que finalmente recibe el turista. Del mismo modo, se establecieron las actividades necesarias para mejorar o mantener los parámetros que se encontraban funcionando. Sin embargo, es necesario resaltar que el funcionamiento de los programas, requiere del direccionamiento estratégico y del funcionamiento correcto del sistema de gestión (Nivel estratégico y táctico)

Con la implementación del Sistema de Gestión para la Sostenibilidad se logró la certificación de la playa La Aguada por parte del ente certificador SGS.

\section{Conclusiones}

Como se ha visto en el desarrollo del documento, la NTS- TS 001-2: 2011, exige el cumplimiento de distintos requisitos. Este diagnóstico de playa La Aguada, se ha realizado para conocer el grado de cumplimiento de estos requisitos. En este caso se evidenció que existían no conformidades que debían ser mejoradas para poder llegar a cumplir la totalidad de requisitos de la NTS- TS 001-2: 2011.

Adicional a los programas específicos para el cumplimiento de los requisitos de la norma, es necesario que dentro del sistema de gestión se trabaje en temas como fortalecimiento de los símbolos culturales, aprovechando el gran potencial que tiene el PNN Utría. Esto permitiría que la playa se constituya en un lugar con identidad y que la comunidad pueda, a través del rescate de la cultura ancestral, encontrar un punto de encuentro para dejar las diferencias y cohesionarse en búsqueda de propuestas inclusivas y sostenibles.

De igual forma, se debe manejar el riesgo, visto como las posibles fallas del propio sistema, lo que ocasionaría que efectivamente se alcance la sostenibilidad de la Playa. Por lo que se sugiere implementar los requisitos de la NTC 5254, como un elemento adicional, y como parte del sistema de gestión de sostenibilidad.

En este tipo de estudios es vital contar con la participación de los diferentes actores que se encuentran involucrados en el manejo del territorio para que se conviertan en parte del proceso investigativo. De esta manera, se podrán obtener resultados que aporten de alguna $\mathrm{u}$ otra forma a la realidad del entorno donde se realiza la investigación y permita el mejoramiento de su calidad de vida. Deben ser los actores que se encuentran en el territorio quienes desarrollen las actividades propuestas en los programas. Además, ellos deben adoptar formas permanentes de conducta que incluyan parámetros de sostenibilidad, conciencia ambiental y social, para que efectivamente el área delimitada como destino turístico sostenible logre ser un lugar con las condiciones de calidad y de respeto en las diferentes dimensiones del desarrollo sostenible.

El acompañamiento de las universidades y profesionales en áreas de ingeniería ambiental, biología o afines a este tipo de procesos de 
construcción colectiva es esencial por el elevado componente técnico de las normas.

\section{Referencias}

Cifuentes, M. (1992). Determinación de Capacidad de Carga Turística en Áreas Protegidas. Turrialba, Costa Rica: Centro Agronómico Tropical de Investigación y Enseñanza Catie.

Ministerio de Comercio, Industria y Turismo. (2011). Documento de Política de Playas Turísticas: Lineamientos Sectoriales. Bogotá D.C.: Ministerio de Comercio, Industria y Turismo.

Ministerio de Comercio, Industria y Turismo. (2014). Plan Sectorial de Turismo 2011-2014, "Turismo: factor de prosperidad para Colombia”. Bogotá D.C.: Ministerio de Comercio, Industria y Turismo.

Ministerio de Comercio, Industria y Turismo. (2014). Plan Sectorial de Turismo 2014 - 2018. "Turismo para la construcción de la paz”. Bogotá D.C.: Ministerio de Comercio, Industria y Turismo.

Gutiérrez-Fernadez, L. F (2013). Funciones de valor para construir un indice de sostenibilidad para la evaluación de áreas naturales con uso turistico. Revista de Tecnología. Bogotá-Colombia, Volumen 12, No. 1, pp 110- 117.
Gutiérrez-Fernadez, L. F et al. (2014).Diseño del sistema de gestión sustentable de playa Grande en el corregimiento de Taganga, Santa Marta - Magdalena, Colombia. Revista de Tecnología. Bogotá - Colombia, Vol. 13, No. 2. 131- 154

Ochoa, G. I. (2008). Turismo en la Amazonia. En G. O. Zuluaga, Turismo en la Amazonia: Entre el desarrollo convencional y las alternativas ambientales amigables (pág. 232). Bogotá: Editora Guadalupe Ltda.

UAESPNN. (2009). Parque Nacional Natural Utría, Plan de Manejo 2005-2009. Parques Nacionales Naturales de Colombia, Proyecto Parques del Pacífico

UPME. (2005). Atlas de radiación solar de Colombia. Ministerio de Minas y Energía - Colombia.

Saucedo, D., \& Torres, A. (1996). Tesis de Grado: Evaluación Organizativa de los Servicios Prestados al Turista en el Corregimiento de Taganga. Santa

Marta: Universidad del Magdalena. Programa de Administración Agropecuaria.

Vásquez, M. A. (2005). Estimación de la Capacidad de Carga Turística en los Senderos de la Reserva Natural Volcán Mombacho, Granada. Managua: Universidad Nacional Agraria.

WWF. (1999). Capacidad de Carga Turística de las Áreas de Uso Público del Monumento Nacional Guayabo. Costa Rica. Costa Rica: WWF Centroamérica. 
ANEXO 1

Matriz de identificación, priorización y calificación de impactos

\section{MATRIZ DE IDENTIFICACION, PRIORIZACION Y CALIFICACIÓN DE IMPACTOS}

\begin{tabular}{|cccccccccc}
\multicolumn{10}{c}{ Zona de la playa La Aguada } \\
Actividades
\end{tabular}

Durabilidad del impacto

Largo plazo más de 10 años

5
Medio tiempo: a 5 años

3
Corto plazo: a 2 años

1

\section{Reversibilidad}

No irreversible 5
Parcialmente reversible

3
Reversible

Afectación a la dimensión ambiental en cada uno de sus componentes (flora, fauna, suelo, aire, agua)

\begin{tabular}{ccc} 
Alta & Media & Baja \\
5 & 3 & 1 \\
\hline
\end{tabular}

Afectación a la dimensión sociocultural

Afecta a poblaciones vulnerables: niños, niñas, tercera edad, entre otros.

$$
5
$$

Afecta mayor número de personas
Afecta a muy pocas personas

\begin{tabular}{ccc}
5 & 3 & 1 \\
\hline
\end{tabular}

Afectación a la dimensión económica

Se pierden puestos de trabajo Los ingresos disminuyen altamente Los ingresos disminuyen poco 


\section{POSIBLES IMPACTOS}

- Pérdida de biodiversidad

- Contaminación por basura

- Contaminación visual

- Contaminación del suelo

- Contaminación por aguas residuales

- Contaminación del mar por combustibles

- Contaminación del mar por lubricantes

- Ruido por presencia de visitantes

- Contaminación auditiva

- Modificaciones en el comportamiento y distribución de la fauna

- Deterioro y perdida de cobertura coralina

- Afectación del sustrato coralino por embarcaciones

- Reducción del recurso pesquero

\section{ACTIVIDADES ECOTURÍSTICAS}

- Interpretación Ambiental

- Senderismo submarino, acuático y terrestre

- Observación de fauna y flora

- Observación de aves

- Avistamiento de ballenas

- Observación de tortugas (caguama)

- Buceo (plataforma)

- Careteo (plataforma)

- Canotaje y entrada al estero hasta el centro de interpretación

- Fotografía

- Video

- Sendero de interpretación estero grande

- Investigación

- Educación ambiental

- Contacto cultural

- Adquisición de artesanías (se va a implementar una ecotienda)

- Tomar el sol 
